\title{
Outcomes of stereotactic radiosurgery for foramen magnum meningiomas: an international multicenter study
}

\author{
Gautam U. Mehta, MD,, Georgios Zenonos, MD, ${ }^{2}$ Mohana Rao Patibandla, MCh, ${ }^{1}$ \\ Chung Jung Lin, MD, ${ }^{3}$ Amparo Wolf, MD, PhD, ${ }^{4}$ Inga Grills, MD, ${ }^{5}$ David Mathieu, MD, ${ }^{6}$ \\ Brendan McShane, BS, ${ }^{7}$ John Y. Lee, MD, ${ }^{7}$ Kevin Blas, MD, ${ }^{5}$ Douglas Kondziolka, MD, ${ }^{4}$ \\ Cheng-Chia Lee, MD, ${ }^{8}$ L. Dade Lunsford, MD, ${ }^{2}$ and Jason P. Sheehan, MD, PhD ${ }^{1}$
}

${ }^{1}$ Department of Neurological Surgery, University of Virginia Medical Center, Charlottesville, Virginia; ${ }^{2}$ Department of Neurological Surgery, University of Pittsburgh Medical Center, Pittsburgh; ${ }^{7}$ Department of Neurosurgery, University of Pennsylvania, Philadelphia, Pennsylvania; Departments of ${ }^{3}$ Neuroradiology and ${ }^{8}$ Neurosurgery, Neurological Institute, Taipei Veterans General Hospital, Taipei, Taiwan, Republic of China; ${ }^{4}$ Department of Neurosurgery, New York University Langone Medical Center, New York, New York; ${ }^{5}$ Department of Radiation Oncology, Beaumont Health System, Royal Oak, Michigan; and ${ }^{6}$ Division of Neurosurgery, Université de Sherbrooke, Centre de recherche du CHUS, Sherbrooke, Quebec, Canada

OBJECTIVE Meningiomas are the most common benign extramedullary lesions of the foramen magnum; however, their optimal management remains undefined. Given their location, foramen magnum meningiomas (FMMs) can cause significant morbidity, and complete microsurgical removal can be challenging. Anterior and anterolateral FMMs carry greater risks with surgery, but they comprise the majority of these lesions. As an alternative to resection, stereotactic radiosurgery (SRS) has been used to treat FMMs in small case series. To more clearly define the outcomes of SRS and to delineate a rational management paradigm for these lesions, the authors analyzed the safety and efficacy of SRS for FMM in an international multicenter trial.

METHODS Seven medical centers participating in the International Gamma Knife Research Foundation (IGKRF) provided data for this retrospective cohort study. Patients who were treated with Gamma Knife radiosurgery and whose clinical and radiological follow-up was longer than 6 months were eligible for study inclusion. Data from pre- and postSRS radiological and clinical evaluations were analyzed. Stereotactic radiosurgery treatment variables were recorded. RESULTS Fifty-seven patients ( 39 females and 18 males, with a median age of 64 years) met the study inclusion criteria. Thirty-two percent had undergone prior microsurgical resection. Patients most frequently presented with cranial neuropathy (39\%), headache (35\%), numbness (32\%), and ataxia (30\%). Median pre-SRS tumor volume was $2.9 \mathrm{~cm}^{3}$. Median SRS margin dose was 12.5 Gy (range 10-16 Gy). At the last follow-up after SRS, $49 \%$ of tumors were stable, $44 \%$ had regressed, and $7 \%$ had progressed. Progression-free survival rates at 5 and 10 years were each $92 \%$. A greater margin dose was associated with a significantly increased likelihood of tumor regression, with $53 \%$ of tumors treated with $>12$ Gy regressing. Fifty-two percent of symptomatic patients noted some clinical improvement. Adverse radiation effects were limited to hearing loss and numbness in 1 patient $(2 \%)$.

CONCLUSIONS Stereotactic radiosurgery for FMM frequently results in tumor control or tumor regression, as well as symptom improvement. Margin doses $>12$ Gy were associated with increased rates of tumor regression. Stereotactic radiosurgery was generally safe and well tolerated. Given its risk-benefit profile, SRS may be particularly useful in the management of small- to moderate-volume anterior and anterolateral FMMs.

https://thejns.org/doi/abs/10.3171/2017.3.JNS163008

KEY WORDS foramen magnum; Gamma Knife radiosurgery; meningioma; radiation; stereotactic radiosurgery

ABBREVIATIONS ARE = adverse radiation effect; FMM = foramen magnum meningioma; GKRS = Gamma Knife radiosurgery; SRS = stereotactic radiosurgery . SUBMITTED December 1, 2016. ACCEPTED March 10, 2017.

INCLUDE WHEN CITING Published online September 1, 2017; DOI: 10.3171/2017.3.JNS163008. 
$\mathrm{F}$ ORAMEN magnum meningiomas (FMMs) represent only $2 \%-3 \%$ of all meningiomas but nearly $75 \%$ of all benign extramedullary lesions at the foramen magnum. ${ }^{1,6,11,20,24}$ While meningiomas are typically slowgrowing neoplasms, tumor progression in this location can cause significant morbidity due to brainstem compression and/or lower cranial nerve palsy. Given the overall infrequency of FMMs, treatment outcomes and optimal management paradigms remain poorly defined.

For several decades, resection has been the treatment of choice for these tumors. While resection of posterior FMMs can often be performed with minimal morbidity, resection of anterior and anterolateral lesions is complicated by a limited surgical corridor and critical intervening neurovascular structures. Tumors at these anterior and anterolateral locations represent more than $90 \%$ of all FMMs. ${ }^{8,13,23}$ The evolution of far lateral and transcondylar skull base techniques has allowed for more extensive resection of a greater proportion of these tumors while minimizing morbidity. ${ }^{1-3,7,9,15,21}$ Despite these advantages, FMMs remain challenging lesions to treat with microsurgery.

Since the 1990s, stereotactic radiosurgery (SRS) has been described as an alternative therapy for FMMs., ${ }^{4}$, $17,19,25$ Given the infrequency of these lesions, only small case series have been reported to date ( $\leq 24$ cases). To more clearly define the role of SRS in the optimal management of these lesions, we performed an international multicenter study on the outcome of Gamma Knife radiosurgery (GKRS) for FMMs.

\section{Methods}

\section{Patients and Tumors}

Patients who had been treated with GKRS for FMMs between 1990 and 2016 were identified within institutional review board-approved retrospective databases at 7 institutions participating in the International Gamma Knife Research Foundation. These institutions included the University of Virginia Medical Center (13 patients), University of Pittsburgh Medical Center (27 patients), Taipei Veterans General Hospital (6 patients), New York University Langone Medical Center (5 patients), Beaumont Health System (3 patients), Université de Sherbrooke (2 patients), and University of Pennsylvania (1 patient). Individual patient data were de-identified and pooled for analyses.

A specific inclusion criterion was either a histologically confirmed WHO Grade I meningioma or a tumor presumed to be a Grade I meningioma based on clinical history and neuroimaging studies. Tumors were considered to be FMMs if the insertion zone was mainly located within the following limits of the foramen magnum, as previously described: ${ }^{3} 1$ ) anteriorly, the lower third of the clivus and the upper limit of the body of C-2;2) laterally, the jugular tubercle and the upper limit of the C-2 lamina; and 3 ) posteriorly, the anterior edge of the squamous occipital bone and the $\mathrm{C}-2$ spinous process.

Tumor location was defined using the classification schema described by George and colleagues: ${ }^{3}$ 1) anterior, insertion on both sides of the anterior midline; 2) antero- lateral, insertion between the anterior midline and the dentate ligament; and 3) posterior, insertion posterior to the dentate ligament.

Patients with less than 6 months of imaging follow-up were excluded.

\section{Radiosurgical Approach}

Single-session GKRS was performed as previously described. ${ }^{19}$ In brief, frame-based stereotaxy was performed using a Leksell Model G frame (Elekta AB) and thin-slice MRI. It was important to lower the frame's base ring to approximately the level of C-2 to adequately treat the caudal extent of some tumors. Computed tomography was frequently used with MRI given that a fusion of the 2 modalities often allowed for optimal imaging of the skull base soft tissue and bony structures. Gamma Knife radiosurgery was then planned by a multidisciplinary team including a neurosurgeon, a radiation oncologist, and a medical physicist. Maximal point spinal cord and brainstem doses were kept below 12 Gy when possible. Radiosurgical parameters, including the margin and maximum radiation dose, isodose line, tumor volume within the prescription isodose, and number of isocenters, were recorded.

\section{Follow-Up}

Patients were typically followed up with MRI at 6-month intervals for the first 2 years and annually thereafter. Tumor progression was defined as changes in overall dimensions that would lead to an increase in volume $>10 \%$ of the pretreatment volume. Tumor response was defined as a decrease in volume $>10 \%$ of the pretreatment volume. ${ }^{18}$ Patients were followed up clinically for neurological sequelae including adverse radiation effects (AREs). These were defined to include cranial neuropathy and/or brainstem-related findings, signs, and symptoms, such as brainstem edema, numbness, or weakness, that were determined to be unrelated to tumor growth. Adverse radiation effects were classified as asymptomatic, symptomatic and temporary, and symptomatic and permanent.

\section{Statistical Analyses}

Primary outcome data (tumor control and regression) were evaluated using Kaplan-Meier analysis to determine progression-free survival from the time of treatment. The effect of preoperative and treatment variables on therapeutic outcome was analyzed using a Cox proportional hazards model by Breslow's method. Multivariate analyses were performed if more than 10 events were recorded and included variables with a 2 -sided $\mathrm{p}$ value $<0.10 .^{14}$ Statistical significance was determined using a 2-sided $\mathrm{p}$ value $<0.05$. Analyses were performed using the R software (version 3.11, The R Foundation for Statistical Computing).

\section{Results}

\section{Patient Characteristics}

Among the 7 participating institutions, 62 patients were 
identified with FMMs that had been treated with singlesession SRS. Five patients were excluded because they either had a follow-up $<6$ months (4 patients) or lacked imaging follow-up (1 patient), resulting in 57 patients available for analyses. Median age was 64 years (range $30-83$ years), and most patients (67\%) were female (Table 1). A minority of patients (32\%) had undergone prior tumor resection a median of 24 months before SRS. Stereotactic radiosurgery was performed for residual tumor in 7 patients (39\%) and recurrent tumor in 11 patients (61\%). Only 2 patients (4\%) had undergone fractionated radiation therapy before SRS. Forty-two patients (74\%) presented with symptomatic tumors, whereas the rest were asymptomatic. The most common presenting symptoms or signs were cranial nerve deficits (39\%), headache (35\%), numbness $(32 \%)$, and ataxia $(30 \%)$. A proportion of cranial nerve deficits $(45 \%-100 \%)$ were related to a prior resection (Table 1). Six patients (10\%) had hydrocephalus prior to radiosurgery, with 5 requiring shunts.

\section{Tumor Characteristics}

Among the 57 tumors, 10 (18\%) were located anteriorly, $39(68 \%)$ anterolaterally, and $8(14 \%)$ posteriorly. Ten tumors $(18 \%)$ were located in the midline. Pretreatment volume was available for 56 tumors with a median volume of $2.9 \mathrm{~cm}^{3}$. Median clinical follow-up was 53 months (range 6-196 months), while the median radiological follow-up was 36 months (range 6-196 months).

\section{Radiosurgical Outcomes}

Stereotactic radiosurgery procedural characteristics are summarized in Table 2. At the last follow-up after SRS, 28 tumors (49\%) remained stable, $25(44 \%)$ decreased in size, and $4(7 \%)$ increased in size (Fig. 1). Thus, total tumor control (stable or regressed tumor) was achieved in $93 \%$ of the patients. By Kaplan-Meier analysis, progression-free survival at 2, 5, 8 , and 10 years was $100 \%, 92 \%, 92 \%$, and $92 \%$, respectively (Fig. 2A). Analyses after 10 years were limited by the number of patients at risk $(\leq 5)$. Pretreatment variables were analyzed with univariate analyses to determine their effect on tumor progression; none of the tested variables predicted a worse response (Table 3). Multivariate analyses were not performed given the paucity of events. Of the 4 patients with growing tumors, 2 were treated with repeat SRS, 1 was treated with resection, and 1 is being observed.

Median time to tumor regression was 16 months (range 6-55 months) in the 25 patients with a radiosurgical response. According to Kaplan-Meier analysis, the cumulative proportion with regressing tumors at 2, 5, 8, and 10 years was 43\%, 54\%, 54\%, and 69\%, respectively (Fig. 2B). Univariate analyses assessing the relationship between pretreatment variables and tumor regression revealed that an increasing margin dose significantly predicted tumor regression ( $p=0.04$; Table 4). A multivariate model including age and margin dose confirmed margin dose as the sole predictor of regression $(p=0.04)$. A statistically significant threshold for margin dose was not identified. However, of the 27 patients who received $\leq 12 \mathrm{~Gy}, 3$ (11\%) had tumor progression and $9(33 \%)$ had tumor regression.
TABLE 1. Baseline characteristics of 57 patients with FMMs treated with GKRS

\begin{tabular}{lc}
\hline \multicolumn{1}{c}{ Variable } & Value \\
\hline Median age at treatment in yrs (range) & $64(30-83)$ \\
\hline No. of females & $39(67 \%)$ \\
\hline Patients w/ prior resection & $18(32 \%)$ \\
\hline $\begin{array}{l}\text { Median time from prior resection to GKRS in } \\
\text { mos (range) }\end{array}$ & $24(2-260)$ \\
\hline Patients w/ prior radiation therapy & $2(4 \%)$ \\
\hline Median KPS score (range) & $90(40-100)$ \\
\hline Symptomatic at presentation & $42(74 \%)$ \\
\hline Presenting symptoms or signs & $20(35 \%)$ \\
\hline Headache & $17\left(2^{*}\right)(30 \%)$ \\
\hline Ataxia & $12\left(3^{*}\right)(21 \%)$ \\
\hline Weakness & $18\left(3^{*}\right)(32 \%)$ \\
\hline Numbness & $2\left(2^{*}\right)(4 \%)$ \\
\hline CN V & $3\left(2^{*}\right)(5 \%)$ \\
\hline CN VI & $3\left(2^{*}\right)(5 \%)$ \\
\hline CN VII & $7\left(2^{*}\right)(12 \%)$ \\
\hline CN VIII & $11\left(5^{*}\right)(19 \%)$ \\
\hline CN IXIX & $7\left(3^{*}\right)(12 \%)$ \\
\hline CN XI & $6\left(3^{*}\right)(10 \%)$ \\
\hline CN XII & $6(10 \%)$ \\
\hline Hydrocephalus & $5(9 \%)$ \\
\hline Prior shunt & $10(18 \%)$ \\
\hline Tumor location & $39(68 \%)$ \\
\hline Anterior & $8(14 \%)$ \\
\hline Anterolat & $10(18 \%)$ \\
\hline Posterior & \\
\hline Midline & \\
\hline Median pre-SRS tumor vol in cm ${ }^{3}($ range) $\dagger$ \\
\hline CNadiological FU in mos (range)
\end{tabular}

In contrast, of the 30 patients who received $>12 \mathrm{~Gy}, 1$ (3\%) had tumor progression and $16(53 \%)$ had tumor regression (Fig. 3).

Among the 42 patients with pre-SRS symptoms, 22 (52\%) had some improvement of their symptoms and none had worsened pretreatment symptoms. Headache improved in 17 of 20 patients with pre-SRS headache, numbness improved in 7 of 18 patients with pre-SRS numbness,

TABLE 2. Characteristics of GKRS for 57 patients with FMMs

\begin{tabular}{lc}
\hline \multicolumn{1}{c}{ Variable } & Median (range) \\
\hline Margin dose in Gy & $12.5(10-16)$ \\
\hline Maximal dose in Gy & $25(14-43)$ \\
\hline Isodose line in \% & $50(30-80)$ \\
\hline Isocenters & $9(2-28)$ \\
\hline
\end{tabular}



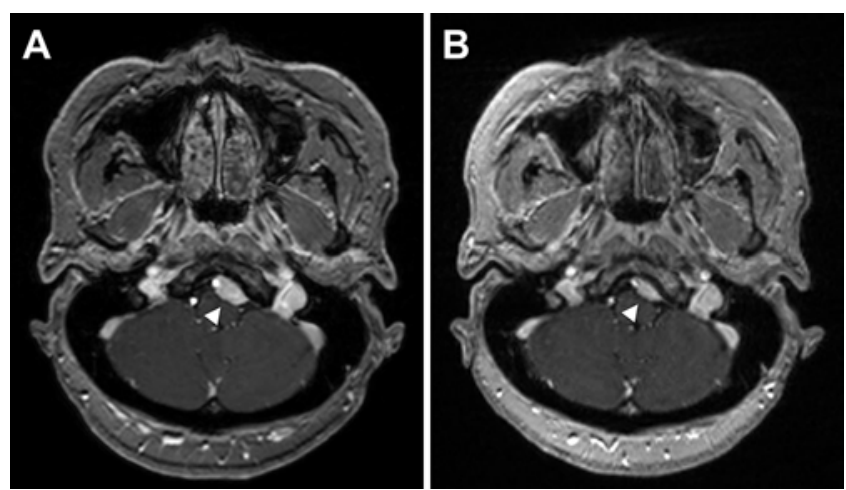

FIG. 1. Postcontrast T1-weighted MR images obtained in a 61-year-old patient with an FMM treated with GKRS. A: Pretreatment imaging revealed an incidentally discovered anterolateral FMM (arrowhead) with a total volume of $1.8 \mathrm{~cm}^{3}$. B: Six months after SRS (14 Gy), imaging revealed a significant decrease in lesion size (arrowhead) with no AREs.

ataxia improved in 5 of 17 patients with pre-SRS ataxia, weakness improved in 4 of 12 patients with pre-SRS weakness, and cranial nerve deficits improved in 4 of 22 patients with pre-SRS deficits.

\section{Adverse Radiation Effects}

Stereotactic radiosurgery for FMM was generally well tolerated. One patient (2\%) experienced a potential ARE that was classified as symptomatic and permanent. This 60 -year-old man presented with partial hearing loss and was treated for a $0.4-\mathrm{cm}^{3}$ anterior FMM that had not been previously treated with surgery or radiation. Stereotactic radiosurgery was performed with a margin dose of $12 \mathrm{~Gy}$ (maximum dose $30 \mathrm{~Gy}$ ). Maximum brainstem dose was 8.2 Gy (mean $1.8 \mathrm{~Gy}$ ) and maximum cochlear dose was $0.5 \mathrm{~Gy}$ (mean $0.4 \mathrm{~Gy}$ ). Thirty-seven months after radiosurgery, the patient experienced worsened hearing loss and new left-sided upper- and lower-extremity numbness. The tumor remained stable in volume through 60 months of radiological follow-up. Magnetic resonance imaging did not demonstrate evidence of T2 signal change in the brainstem or spinal cord. Neither the hearing loss nor the numbness in this patient could be determined to be definitively related to radiosurgery.

\section{Discussion}

\section{Foramen Magnum Meningiomas}

Meningiomas are the most common benign extramedullary lesions of the foramen magnum.11,20,24 Given the proximity of these lesions to the lower cranial nerves, the vertebral arteries and their branches, and the medulla, growth of these lesions can cause significant morbidity. Historically, the primary treatment modality for FMM has been microsurgical resection, including for anterior and anterolateral lesions, which are more challenging to resect and account for approximately $90 \%$ of FMMs. ${ }^{8,13,23}$ Microsurgery results in complete resection in 63\%-96\% of patients in large series from experienced centers. ${ }^{2,8,13,16}$ Despite these results, the attendant risks of FMM resection can be significant, with morbidity occurring after
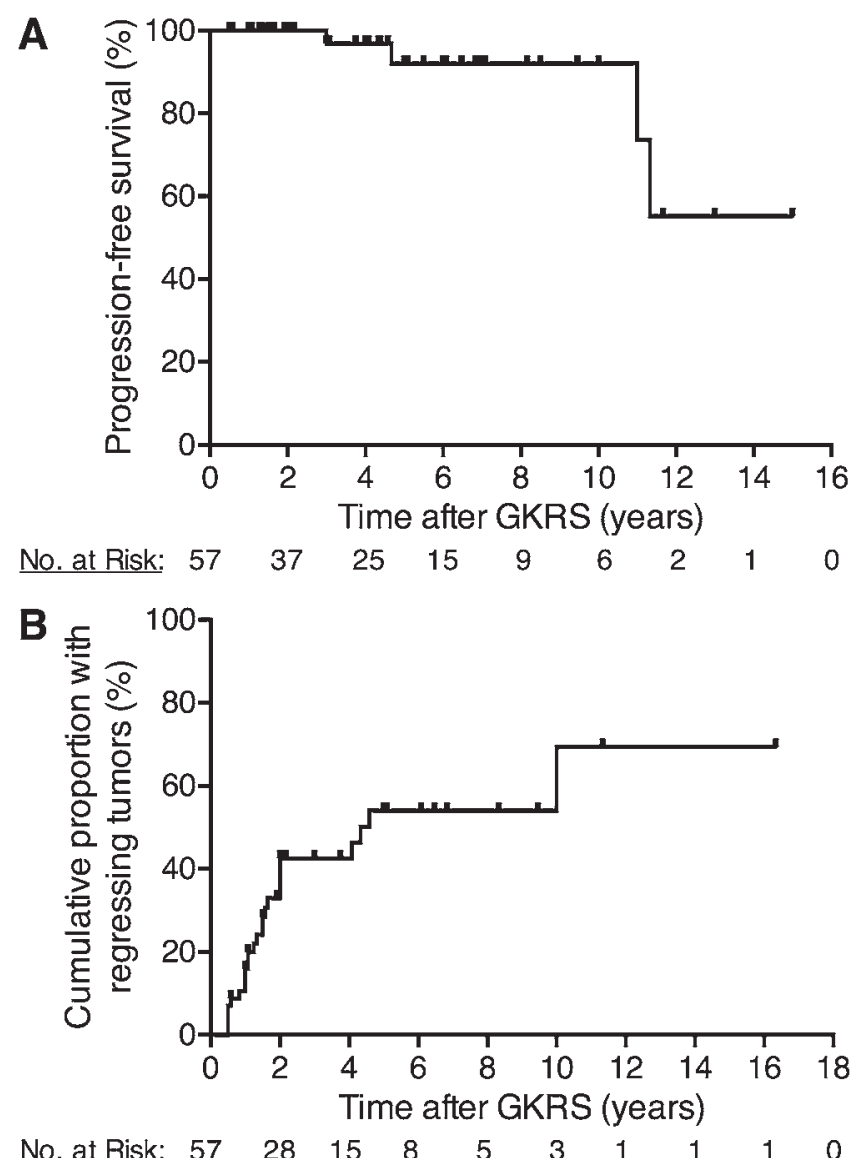

FIG. 2. Kaplan-Meier analyses of tumor progression and regression after SRS for FMMs. A: In analyzing tumor growth, progression-free survival at $2,5,8$, and 10 years after SRS was found to be $100 \%, 92 \%, 92 \%$, and $92 \%$, respectively. B: In analyzing tumor regression, the cumulative proportion of patients with regressing tumors at 2, 5, 8, and 10 years after SRS was found to be $43 \%, 54 \%, 54 \%$, and $69 \%$, respectively.

$7.5 \%-32 \%$ of operations and deaths occurring after $0 \%-$ $7.5 \%$ of operations in these studies. ${ }^{2,8,13,16}$

\section{Stereotactic Radiosurgery}

Given the risk of complications associated with resection, minimally invasive therapeutic strategies have been considered to be beneficial in carefully selected patients. Stereotactic radiosurgery is generally delivered as a single-session focused approach for patients. Considering the location of the target and the adjacent critical structures, these cases require accurate and precise radiation delivery. Gamma Knife radiosurgery using a frame-based approach typically affords submillimeter accuracy and precision. Early reports have shown that SRS can be effective for FMM, with tumor control rates of $80 \%$ or more..$^{12,17}$, ${ }^{19,25}$ However, these results have been limited by both the number of subjects and the lack of long-term follow-up. Only 2 reports have included more than 10 patients. Zenonos and colleagues analyzed the results in 21 patients with FMMs treated with GKRS (median tumor volume $4.8 \mathrm{~cm}^{3}$, median radiation dose $13 \mathrm{~Gy}$ ) and observed $100 \%$ tumor control at the last follow-up (median 47 months). ${ }^{25}$ 
TABLE 3. Univariate analyses of the relationship between preoperative variables and tumor progression after GKRS for FMMs

\begin{tabular}{lc}
\hline Variable & $p$ Value \\
\hline Age & 0.24 \\
\hline Sex & 0.95 \\
\hline Prior resection & 0.12 \\
\hline Tumor vol & 0.29 \\
\hline Margin dose & 0.18 \\
\hline Maximum dose & 0.53 \\
\hline
\end{tabular}

Symptom improvement was recorded in 10 of 17 symptomatic patients. The North American Gamma Knife Consortium (currently the International Gamma Knife Research Foundation) reported the results of SRS for posterior fossa meningiomas in 2015. ${ }^{17}$ This study included 18 patients with FMMs; however, the results based on individual tumor locations, including the foramen magnum, were not detailed.

\section{The Current Study}

In the present study, we aimed to address these limitations by using an international multicenter approach, which resulted in the largest study of SRS for FMM to date with 57 patients meeting the inclusion criteria (median radiological follow-up 36 months). Analysis showed that SRS for FMM is durable, with $92 \%$ progression-free survival at 10 years after treatment (Fig. 2A). Greater tumor volume was not a factor predictive of growth after SRS; however, relatively small- to moderate-volume tumors were treated in this study (median volume $2.9 \mathrm{~cm}^{3}$, range $0.4-17 \mathrm{~cm}^{3}$ ). Increasing margin dose was found to predict tumor regression (Table 4). Because of the limited numbers treated at each specific margin dose, a statistically significant threshold was not identified, but the majority of tumors treated with a margin dose $>12$ Gy regressed (53\%; Fig. 3).

In general, SRS for FMM offers clinical benefit. Symptom improvements were noted in the majority of symptomatic patients (52\%) who were treated with SRS. Most commonly, headache improved (17 of 20 patients). However, cranial neuropathies were less likely to improve (4 of 22 patients), but they also did not typically worsen. Conversely, AREs were uncommon in this series (2\%). No definitive brainstem or spinal cord toxicity was identified.

\section{Study Limitations}

This study was limited primarily by its retrospective design. Despite the international multicenter approach, FMMs are uncommon tumors; therefore, the number of subjects limited robust statistical analyses. Additionally, there is overlap between some patients in this study and those from prior single-center reports from the participating sites. However, data for all patients accrued were updated for the current study. A future multicenter prospective study will be needed to more definitively determine the optimal management approach for patients with FMM.

The outcomes of this study were the result of radiosur-
TABLE 4. Univariate analyses of the relationship between preoperative variables and tumor regression after GKRS for FMM

\begin{tabular}{ll}
\hline Variable & $p$ Value \\
\hline Age & 0.08 \\
\hline Sex & 0.70 \\
\hline Prior resection & 0.49 \\
\hline Tumor vol & 0.15 \\
\hline Margin dose & $0.04^{*}$ \\
\hline Maximum dose & 0.39 \\
\hline
\end{tabular}

* HR 1.41 (95\% Cl 1.02-1.94).

gical plans created over 3 decades; therefore, dose plan reconstructions for early cases are not feasible. The dose planning performed in Gamma Knife does not typically contour the brainstem in its entirety. Thus, dose-volume information for the brainstem could not readily be obtained. Doses of 12 or 12.5 Gy to a broader meningiomabrainstem interface can also likely be tolerated. In the Quantitative Analysis of Normal Tissue Effects in the Clinic (QUANTEC) report, the authors concluded that a single-session maximum radiosurgical dose of $12.5 \mathrm{~Gy}$ to the brainstem is associated with a $<5 \%$ risk. ${ }^{10}$ Higher doses of 15-20 Gy have been used in patients with other underlying conditions (for example, arteriovenous malformations or metastases) and conveyed a low rate of complications. ${ }^{5,22}$ Regardless, further prospective data using modern radiosurgical planning systems and contouring will be required to determine the brainstem dose-volume constraints for single-session SRS cases.

Finally, the current study does not address the use or value of hypofractionated SRS and its potential role in the management of complex skull base tumors including FMM. This subject will require further investigation,

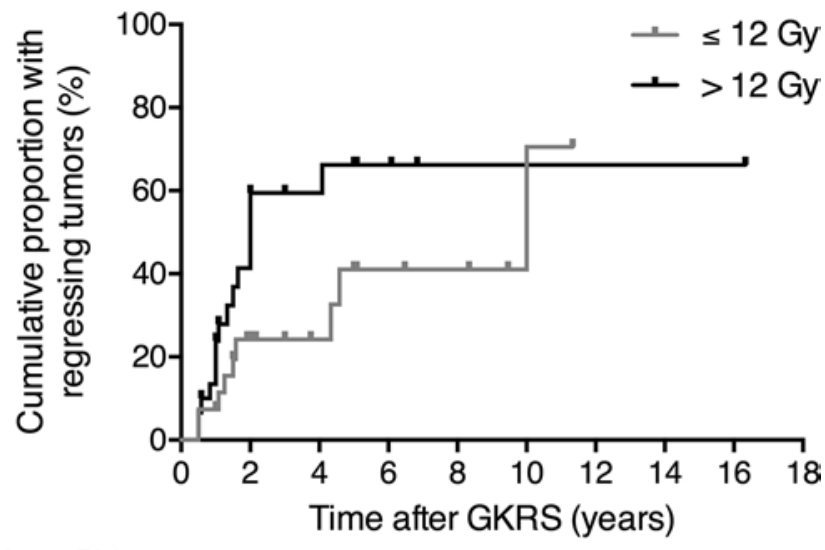

No. at Risk:

$\begin{array}{lllllllllll}\leq 12 \text { Gy } & 27 & 15 & 9 & 5 & 4 & 2 & 0 & 0 & 0 & 0 \\ >12 \text { Gy } & 30 & 13 & 6 & 3 & 1 & 1 & 1 & 1 & 1 & 0\end{array}$

FIG. 3. Kaplan-Meier analysis of tumor regression in patients who received a margin dose $\leq 12 \mathrm{~Gy}$ and those who received $>12 \mathrm{~Gy}$. Although more patients among the group receiving $>12$ Gy demonstrated treatment responses (53\% vs $33 \%$ ), this difference was not statistically significant according to a Cox proportional hazards model $(p=0.07)$. 
particularly as GKRS centers expand the use of hypofractionated approaches in addition to continuing traditional single-session SRS techniques.

\section{Management of FMMs}

The current study demonstrates that single-session SRS can safely achieve durable tumor control in the majority of patients with small to moderate-sized FMMs. Large tumors with significant and symptomatic brainstem compression will likely continue to require surgery for immediate volume reduction. For small- to moderatevolume tumors and for residual or recurrent tumor after resection, SRS is a valuable therapeutic approach. Among FMMs, posterior lesions have a more favorable resection profile. Regardless, SRS has a clear alternative role in the treatment of symptomatic or growing posterior FMM, especially in patients who are elderly, are on anticoagulants, or have an otherwise appreciable risk with microsurgery.

Anterior and anterolateral FMMs comprise the majority of meningiomas at the craniocervical junction. ${ }^{8,13,23}$ With these lesions and based on the best available, albeit imperfect, data from this and other published studies, lower rates of complete resection and greater potential morbidity associated with resection appear to favor a greater role for SRS. For example, in the report on anterior and anterolateral FMMs by Bassiouni et al., mortality and new morbidity rates of $4 \%$ and $8 \%$, respectively, were recorded. ${ }^{2}$ For elderly patients with anterior or anterolateral FMM, those with greater medical risks with microsurgery, and those who prefer a lower-risk therapy, SRS has a favorable risk-benefit profile compared with that for microsurgical resection. For the remaining patients, treatment decisions must be individualized and should factor in radiological findings, symptom burden, and performance status as well as patient preference.

\section{Conclusions}

Single-session SRS represents a safe and highly effective therapeutic option for patients with small- to moderate-volume FMM. Tumor control or tumor regression was achieved in the majority of patients, with most patients experiencing some symptom improvement and/or neurological preservation. These results appeared to be durable well beyond 5 years after radiosurgery. Increased tumor regression was observed with margin doses $>12 \mathrm{~Gy}$. Adverse radiation effects in this location were infrequent. Finally, given its relatively low risk profile, SRS may have particular utility as the primary management for patients with small to moderately sized anterior and anterolateral FMMs.

\section{Acknowledgments}

We appreciate the assistance of Ms. Linda Baxendell with coordination of data for the International Gamma Knife Research Consortium.

\section{References}

1. Arnautović KI, Al-Mefty O, Husain M: Ventral foramen magnum meninigiomas. J Neurosurg 92 (1 Suppl):71-80, 2000
2. Bassiouni H, Ntoukas V, Asgari S, Sandalcioglu EI, Stolke D, Seifert V: Foramen magnum meningiomas: clinical outcome after microsurgical resection via a posterolateral suboccipital retrocondylar approach. Neurosurgery 59:1177-1187, 2006

3. Bruneau M, George B: Foramen magnum meningiomas: detailed surgical approaches and technical aspects at Lariboisière Hospital and review of the literature. Neurosurg Rev 31:19-33, 2008

4. Cheshier SH, Hanft SJ, Adler JR, Chang SD: CyberKnife radiosurgery for lesions of the foramen magnum. Technol Cancer Res Treat 6:329-336, 2007

5. Cohen-Inbar O, Ding D, Chen CJ, Sheehan JP: Stereotactic radiosurgery for deep intracranial arteriovenous malformations, part 1: Brainstem arteriovenous malformations. J Clin Neurosci 24:30-36, 2016

6. Dodge HW Jr, Gottlieb CM, Love JG: Benign tumors at the foramen magnum; surgical considerations. J Neurosurg 13:603-617, 1956

7. Flores BC, Boudreaux BP, Klinger DR, Mickey BE, Barnett SL: The far-lateral approach for foramen magnum meningiomas. Neurosurg Focus 35(6):E12, 2013

8. George B, Lot G, Boissonnet H: Meningioma of the foramen magnum: a series of 40 cases. Surg Neurol 47:371-379, 1997

9. Kano T, Kawase T, Horiguchi T, Yoshida K: Meningiomas of the ventral foramen magnum and lower clivus: factors influencing surgical morbidity, the extent of tumour resection, and tumour recurrence. Acta Neurochir (Wien) 152:79-86, 2010

10. Mayo C, Yorke E, Merchant TE: Radiation associated brainstem injury. Int J Radiat Oncol Biol Phys 76 (3 Suppl):S36-S41, 2010

11. Meyer FB, Ebersold MJ, Reese DF: Benign tumors of the foramen magnum. J Neurosurg 61:136-142, 1984

12. Muthukumar N, Kondziolka D, Lunsford LD, Flickinger JC: Stereotactic radiosurgery for anterior foramen magnum meningiomas. Surg Neurol 51:268-273, 1999

13. Pamir MN, Kiliç T, Özduman K, Türe U: Experience of a single institution treating foramen magnum meningiomas. J Clin Neurosci 11:863-867, 2004

14. Peduzzi P, Concato J, Feinstein AR, Holford TR: Importance of events per independent variable in proportional hazards regression analysis. II. Accuracy and precision of regression estimates. J Clin Epidemiol 48:1503-1510, 1995

15. Pirotte BJM, Brotchi J, DeWitte O: Management of anterolateral foramen magnum meningiomas: surgical vs conservative decision making. Neurosurgery 67 (3 Suppl Operative):ons58-ons70, 2010

16. Samii M, Klekamp J, Carvalho G: Surgical results for meningiomas of the craniocervical junction. Neurosurgery 39:1086-1095, 1996

17. Sheehan JP, Starke RM, Kano H, Barnett GH, Mathieu $\mathrm{D}$, Chiang V, et al: Gamma Knife radiosurgery for posterior fossa meningiomas: a multicenter study. J Neurosurg 122:1479-1489, 2015

18. Snell JW, Sheehan J, Stroila M, Steiner L: Assessment of imaging studies used with radiosurgery: a volumetric algorithm and an estimation of its error. Technical note. J Neurosurg 104:157-162, 2006

19. Starke RM, Nguyen JH, Reames DL, Rainey J, Sheehan JP: Gamma Knife radiosurgery of meningiomas involving the foramen magnum. J Craniovertebr Junction Spine 1:23-28, 2010

20. Stein BM, Leeds NE, Taveras JM, Pool JL: Meningiomas of the foramen magnum. J Neurosurg 20:740-751, 1963

21. Suhardja A, Agur AMR, Cusimano MD: Anatomical basis of approaches to foramen magnum and lower clival meningiomas: comparison of retrosigmoid and transcondylar approaches. Neurosurg Focus 14(6):e9, 2003

22. Trifiletti DM, Lee CC, Kano H, Cohen J, Janopaul-Naylor 
J, Alonso-Basanta M, et al: Stereotactic radiosurgery for brainstem metastases: an international cooperative study to define response and toxicity. Int J Radiat Oncol Biol Phys 96:280-288, 2016

23. Wu Z, Hao S, Zhang J, Zhang L, Jia G, Tang J, et al: Foramen magnum meningiomas: experiences in 114 patients at a single institute over 15 years. Surg Neurol 72:376-382, 2009

24. Yasuoka S, Okazaki H, Daube JR, MacCarty CS: Foramen magnum tumors. Analysis of 57 cases of benign extramedullary tumors. J Neurosurg 49:828-838, 1978

25. Zenonos G, Kondziolka D, Flickinger JC, Gardner P, Lunsford LD: Gamma Knife surgery in the treatment paradigm for foramen magnum meningiomas. J Neurosurg 117:864873,2012

\section{Disclosures}

Dr. Grills reports service on the board of directors for and stock ownership in Greater Michigan Gamma Knife and, through her institution, the receipt of funding for non-study-related research from Elekta. Dr. Lunsford has stock ownership in Elekta AB and is a consultant for Insightec.

\section{Author Contributions}

Conception and design: Sheehan, Mehta. Acquisition of data: Mehta, Zenonos, Patibandla, Lin, Wolf, Grills, Mathieu, McShane, JY Lee, Blas, Kondziolka, CC Lee. Analysis and interpretation of data: Sheehan, Mehta, Lunsford. Drafting the article: Mehta. Critically revising the article: Sheehan, Mehta, Kondziolka,

CC Lee, Lunsford. Reviewed submitted version of manuscript: all authors. Approved the final version of the manuscript on behalf of all authors: Sheehan. Statistical analysis: Mehta. Study supervision: Sheehan.

\section{Correspondence}

Jason P. Sheehan, Department of Neurological Surgery, University of Virginia Medical Center, PO Box 800212, Charlottesville, VA 22908. email: jsheehan@virginia.edu. 